\title{
Testicular sparing surgery in small testis masses: A multinstitutional experience
}

\author{
Andrea B. Galosi ${ }^{1}$, Paola Fulvi ${ }^{1}$, Andrea Fabiani ${ }^{2}$, Lucilla Servi $^{2}$, Alessandra Filosa ${ }^{3}$, Luca Leone ${ }^{1}$, \\ Angelo Marronaro ${ }^{4}$, Enrico Caraceni ${ }^{4}$, Rodolfo Montironi ${ }^{5}$ \\ ${ }^{1}$ Clinica Urologica, Dipartimento Scienze Cliniche e Odontostomatologiche, Università Politecnica delle Marche, \\ AO Ospedale Riuniti, Ancona, Italy; \\ ${ }^{2}$ UOC Urologia Macerata, Area Vasta 3, ASUR Marche, Italy; \\ ${ }^{3}$ UOC Anatomia Patologica, Area Vasta 3, ASUR Marche, Italy; \\ ${ }^{4}$ UOC Urologia, Civitanova Marche, Area Vasta 3, ASUR Marche, Italy; \\ ${ }^{5}$ UOC Anatomia Patologica, Università Politecnica delle Marche, Ancona, Italy.
}

\begin{abstract}
Summary Introduction: The incidence of benign testicular tumors is increasing in particular in small lesion incidentally found at scrotal ultrasonography. Primary aim of this study was to perform radical surgery in malignant tumor. Secondary aim was to verify the efficacy of the diagnostic-therapeutic pathway recently adopted in management of small masses with testis sparing surgery in benign lesions.

Materials and methods: In this multicenter study, we reviewed all patients with single testis lesion less than $15 \mathrm{~mm}$ at ultrasound as main diameter. We applied the diagnostic-therapeutic pathway described by Sbrollini et al. (Arch Ital Urol Androl 2014; 86:397) which comprises: 1) testicular tumor markers, 2) repeated scrotal ultrasound at the tertiary center, 3) surgical exploration with inguinal approach, intraoperative ultrasound, and intraoperative pathological examination. Definitive histology was reviewed by a dedicated uro-pathologist. Results: Twenty-eight patients completed this clinical flowchart. The mean lesion size was $9.3 \mathrm{~mm}$ (range 2.5-15). Testicular tumor markers were normal except in a case. Intraoperative ultrasound was necessary in $8 / 28$ cases. We treated 11/28 (39.3\%) with immediate radical orchiectomy and $17 / 28(60.7 \%)$ with testis-sparing surgery. Definitive pathological results were: malignant tumor in 6 cases (seminoma), benign tumor in 10 cases (5 Leydig tumors, 2 Sertoli tumors, 1 epidermoid cyst, 1 adenomatoid tumor, 1 angiofibro$\mathrm{ma}$ ), benign disease in 11 (8 inflammation with haemorragic infiltration, 2 tubular atrophy, 1 fibrosis), and normal parenchyma in 1 case. We observed a good concordance between frozen section examination and definitive histology. Any malignant tumor was treated conservatively.

Any delayed orchiectomy was necessary based on definitive histology.

Conclusions: The incidence of benign lesions in $60 \%$ of small testis lesions with normal tumor markers makes orchiectomy an overtreatment. Testicular sparing surgery of single testicular nodules below $15 \mathrm{~mm}$ is a safe option, but requires a standardized pathway in diagnosis. Our pathway has shown good reliability and security profile to be applied in a multicenter management for small scrotal masses. Our study has shown the reliability of the diagnostic-therapeutic pathway in the management of single testicular masses. The higher incidence of benign lesions in $60 \%$ of patients makes often orchiectomy an overtreatment.
\end{abstract}

KEY WORDS: Testis neoplasms; Ultrasound; Surgery; Small testis masses.

Submitted 15 November 2016; Accepted 15 December 2016

\section{INTRODUCTION}

Scrotal ultrasound increased detection of small and not palpable testicular lesions. The incidence of benign tumors is clinically relevant in lesions smaller than 15 $\mathrm{mm}(1,2)$. This suggest organ-sparing surgery rather than radical orchiectomy $(3,4)$.

The first step to diagnose testicular cancer is the clinical examination, principally with scrotal palpation, that can give to the physician the first suspect of tumor. But it is possible only for masses which have a large volume. Consequently patients must undergo scrotal ultrasound, that confirms the presence of the neoplasia. The definitive histologic diagnosis of testicular cancer can only be achieved through exploration and inguinal orchiectomy and subsequent histological examination (5). In fact, the gold standard for the treatment of palpable testicular lesions is inguinal exploration and orchiectomy. Meanwhile, the diagnostic-therapeutic pathway for small and not palpable small testicular lesions exclusively identified by ultrasonography is still under debate. According to international guidelines testis-sparing surgery can be performed only in special and selected cases: bilateral testicular tumors (synchronous bilateral testicular tumors or metachronous contralateral tumors) or monorchid patient with normal pre-operative testosterone levels when tumor volume is less than $30 \%$ of testicular volume (2-4). For some years the testis sparing surgery is being practiced with success, with good oncologic out-comes, but there is not currently internationally agreed protocol for management and treatment of small non-palpable testicular masses. Our team published a flow chart including ultrasound diagnosis and surgical management of lesion with maximum diameter of $15 \mathrm{~mm}$ (6). This protocol was established with agreement of pathology, radiology, urology and oncology units. However application of this clinical protocol is not yet published.

Primary aim of this study was to perform radical surgery in all malignant tumor. Secondary aim was to verify the efficacy of the diagnostic-therapeutic pathway recently adopted in management of small masses with testis sparing surgery only in benign lesions. 


\section{Materials AND Methods}

In this multicenter study, we recruited patients with single testicular lesion of $15 \mathrm{~mm}$ as main diameter, based on scrotal ultrasound. We applied the diagnostic-therapeutic pathway described by Sbrollini et al. (6) which comprises: 1) normal testicular tumor markers, 2) repeated scrotal ultrasound at the tertiary center to confirm size, localization and ultrasound characteristics, 3) surgical exploration with inguinal approach using intraoperative ultrasound, and intraoperative pathological examination. The diagnostic-therapeutic pathway recently adopted in management of small masses with testis sparing surgery is summarized in Figure 1.

Definitive histology was reviewed by a dedicated uro-pathologist. After their identification, the nodule was removed and 3 additional biopsies were perfomed in surround normal-looking parenchyma. If the pathologist considered the nodule size with enough amount of tissue, the intraoperative frozen was done, otherwise if not the specimen was sent for definitive histology. In case of benign or doubtful histology on frozen section, the specimen were submitted to definitive exam. Delayed orchiectomy was suggested only if malignant neoplasia was confirmed. In case of testicular intraepithelial neoplasia, patients were addressed to radiotherapy or orchyfuniculectomy. The protocol suggests that small masses $(<8 \mathrm{~mm})$ are sent to the pathologist for histological definitive examination, avoiding the extemporaneous examination, which due to the small volume of the tumor, might subtract material necessary for definitive histological diagnosis. The masses of diameter between 8 and $15 \mathrm{~mm}$ are sent to the pathologist for the extemporaneous histological examination of frozen sections, which determines: histological nature, size and relationship with the surrounding parenchyma. All testicular masses were submitted to definitive histological examination including immunohistochemistry.

\section{RESULTS}

We recruited 28 patients, mean age was 38 years (18-68) and mean lesion size was $9.3 \mathrm{~mm}$ (range 2.5-15). Baseline clinical features are summarized in Table 1 and 2. Oncological testicular markers were normal except in a case who had small increase beta-HCG (9.0). Intraoperative ultrasound was necessary in $8 / 28$ cases to identify the exact cancer location. In particular, 10/28 (36\%) patients had testicular nodules smaller than $8 \mathrm{~mm}$ and 18/28 (64\%) between 8 and $15 \mathrm{~mm}$ (Figure 3-6). Concerning nodules smaller than $8 \mathrm{~mm}, 2 / 10$ underwent definitive histological examination (because of low volume of the sample). Primary aim of this study was to perform radical surgery in malignant tumor. We performed 11
(39.3\%) radical orchiectomy and 17 (60.7\%) testis-sparing procedures. Secondary aim was to verify the efficacy of the diagnostic-therapeutic pathway recently adopted in management of small masses with testis sparing surgery in benign lesions: any delayed orchiectomy was necessary and any radical surgery was performed in benign lesions (Figure 2).

Eight of 10 nodules smaller than $8 \mathrm{~mm}$ underwent frozen section examination. All the 18 nodules between 8 and 15 have been subjected to frozen section histological examina-

Table 2.

Summary of results.

\begin{tabular}{|lc|}
\hline Total patients & $\mathbf{2 8}$ \\
\hline Mean age & $38(18-68)$ \\
\hline Mean lesion size (mm) & $9.3(2.5-15)$ \\
\hline High alfa feto protein & 0 \\
\hline High lattic dehidrogenasi & 0 \\
\hline High beta-human corionic gonadot. & $1(3.6 \%)$ \\
\hline Intraoperative ultrasound & $8(28.6 \%)$ \\
\hline Intraoperative orchiectomy & $11(39.3 \%)$ \\
\hline Delayed orchiectomy & 0 \\
\hline Testis sparing surgery & $17(60.7 \%)$ \\
\hline Malignant tumor & $10(21.4 \%)$ \\
\hline Benign tumor & $11(39.7 \%)$ \\
\hline Benign disease & $1(3.6 \%)$ \\
\hline Normal parenchyma & \\
\hline
\end{tabular}


tion. 19/26 masses subjected to frozen section examination resulted benign tumors: 14/19 of them have been treated conservatively, while 5/19 underwent intraoperative radical orchiectomy. We observed a good concordance between frozen section examination and definitive histology, without any false positive results at frozen section. One of 26 mass resulted as healthy parenchyma. Six of 26 masses resulted as malignant tumors and underwent radical orchiectomy. We have had no cases of benign tumor associated with Testicular Intraepithelial Neoplasia. Definitive pathological results were: malignant tumor in 6 cases (seminoma), benign tumor in 10 cases (5 Leydig tumors, 2 Sertoli tumors, 1 epidermoid cyst, 1 adenomatoid tumor, 1 angiofibroma), benign disease in 11 ( 8 inflammation with hemorragic infiltration, 2 tubular atrophy, 1 fibrosis), and normal parenchyma in 1 case (Table 2).

\section{Discussion}

Our study has shown the reliability of the diagnostic-therapeutic pathway suggested by Sbrollini et al. in the management of single testicular masses below $15 \mathrm{~mm}$. The higher incidence of benign lesions in this group of tumors (60\%) makes often orchiectomy an overtreatment. We observed a complete concordance between frozen section examination and definitive histology regarding malignant cell. Previous experience in high volume centers had same results with dedicated pathologist $(7,8)$, pointing out that in our center there was always a dedicated uro-pathologist too. In our experience the definitive intraoperative diagnosis of non-palpable testicular lesions was always reliable even in very small lesions (< $8 \mathrm{~mm})$. However in case of uncertain diagnosis or small tissue, the definitive histology is recommended. This protocol is useful also for centres were the frozen section is not available: very small lesions could be managed with definitive histology and any subsequent decision can be delayed. In our experience, intraoperative histology on frozen section was possible in 8 of 10 small nodules with diameter less than $8 \mathrm{~mm}$,
Figure 1.

Diagnostic and surgical flow chart for management of not-palpable testis masses modified from Sbrollini et al.(6).

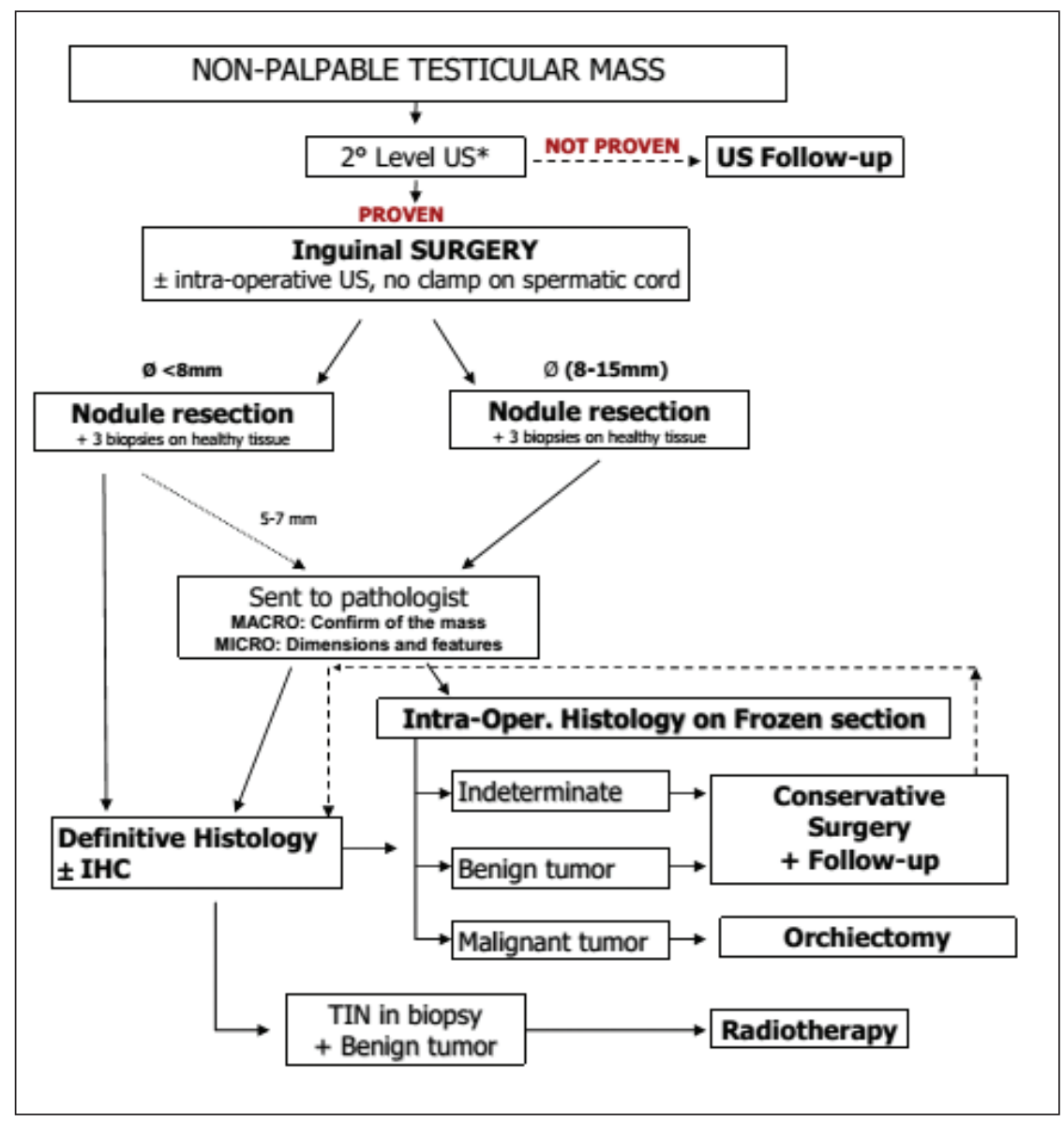

Figure 2.

Oncological results in 28 patients submitted to surgery according to flow chart.

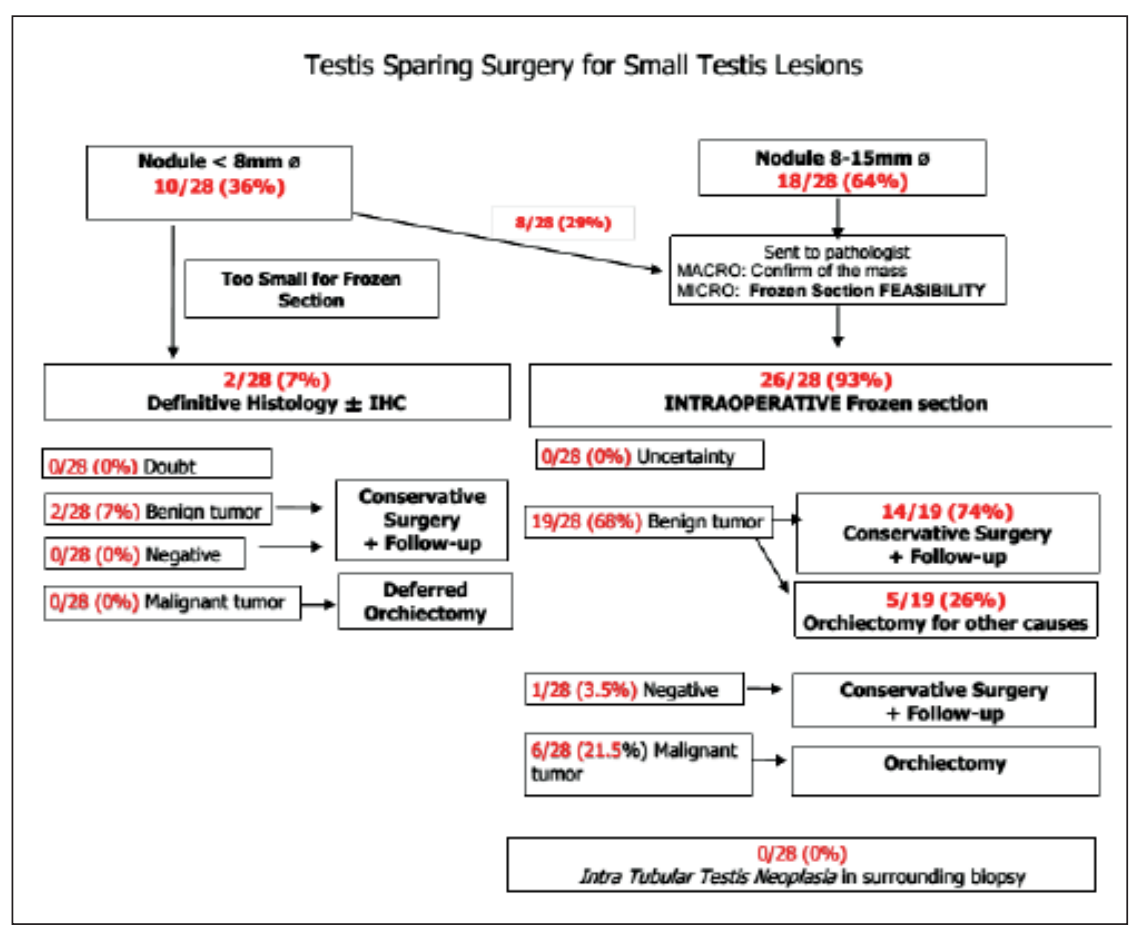


Figure 3.

Small solid hypoechoic lesion $(5 \times 3 \mathrm{~mm}$ ) well defined compared to normal parenchyma: Leydig cell tumor.
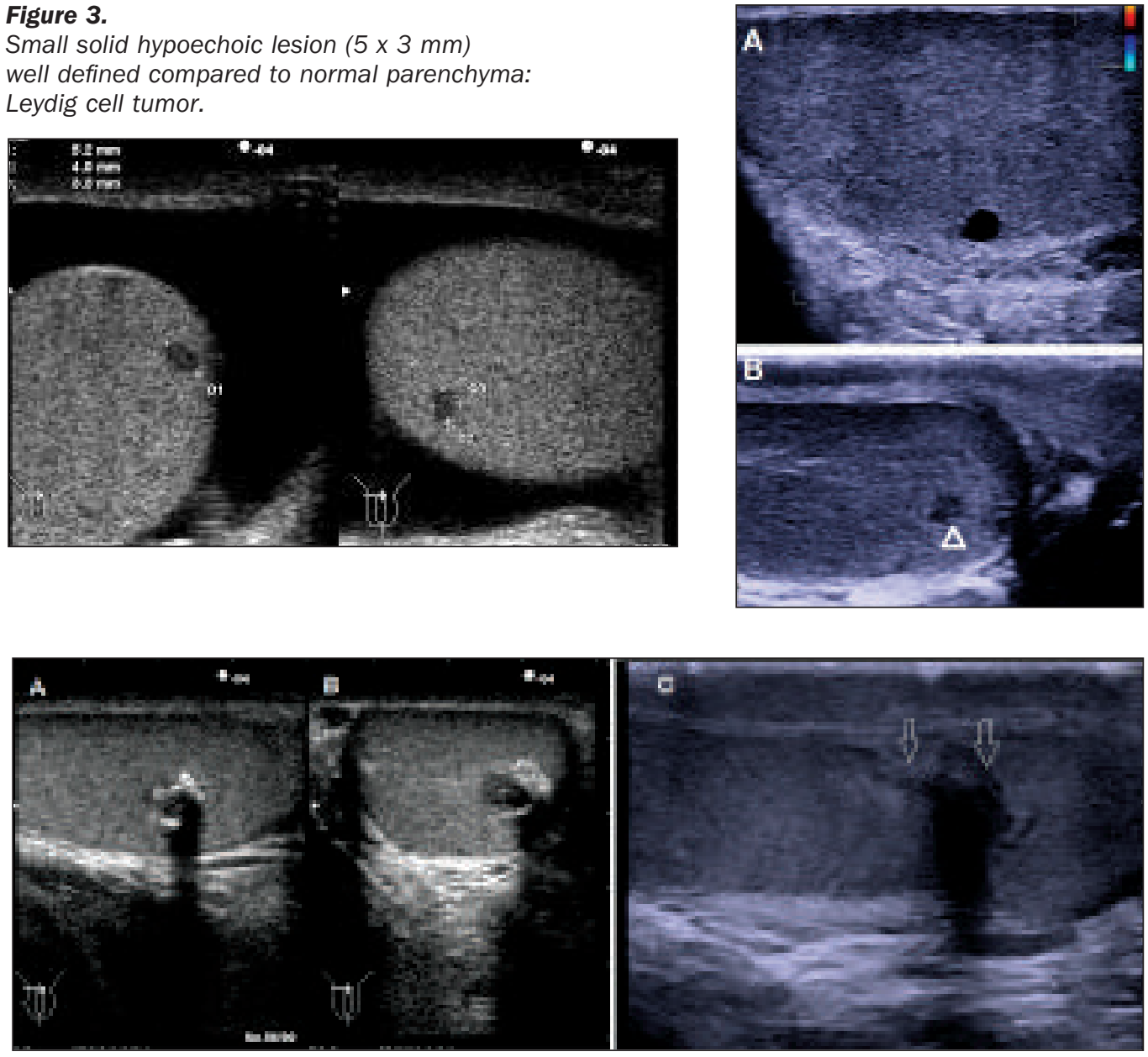

Figure 5.

Epidermoid cyst with circular calcifications (A longitudinal view; $B$ axial). The follow-up ultrasound: 8 years later after testis sparing surgery $(C$, arrows show surgical scar).
Figure 6.

Leydig tumor with irregular bordered and irregular content with very small anechoic lacunas.

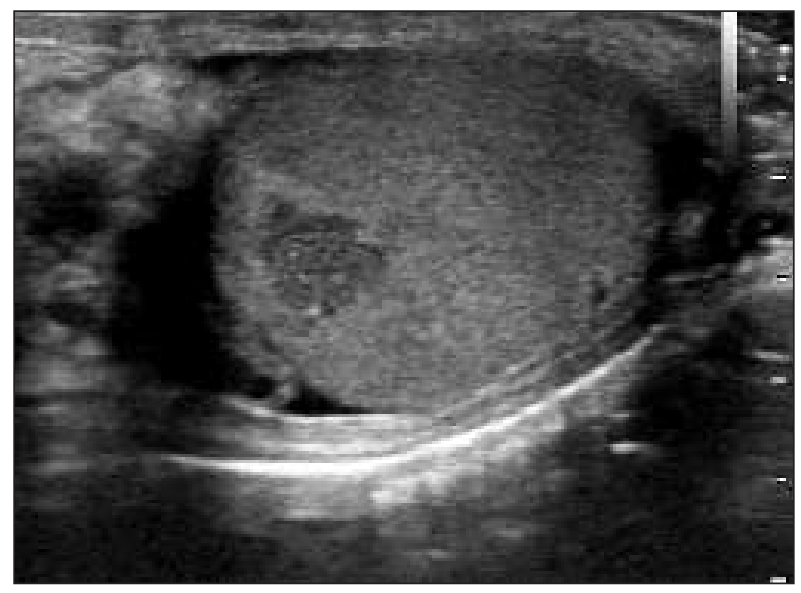

without any false positive results and without wasting tissue for the final histology and ancillary immunohistochemistry. Only 20\% of the specimens were considered unsuitable for frozen section analysis. Unusual malignant tumor has been observed also arising from stromal cells tumors (e.g. malignant Leydig cells tumors, malignant Sertoli cells tumors). The differential diagnosis may be not easy among malignant and benign testis tumor such as Sertoli tumor, Leydig cells tumors, epidermoid cyst, Brenner tumor, adenomas of the ducts. Testis sparing surgery is adopted also in selected cases in children (9). During surgery the surgeon should use loop for optical magnification and use palpation to detect the nodule. In case of soft nodule, were the palpation is not helpful, the intraoperative ultrasound is mandatory. This protocol should be shared in the first instance by the involved specialists, such as the urologist, as an active operator in the operating room, the pathologist, the oncologist and the radiologist. High resolution and high frequency ultrasound (12 MHz) with adequate focusing, fine-tunig and grayscale regulation should be ready available in operating room. Machine with low resolution and frequency $(8 \mathrm{MHz})$ with inadequate tuning are not enough to have a good imaging and could be misleading (10). The patient, before being subjected to this type of approach has to be informed in detail about the various benefits, risks and implications, first of all the possibility of being subjected to a second surgery as soon as the urologist is in possession of the definitive histological result.

\section{Conclusion}

Testicular sparing surgery of single testicular nodules below $15 \mathrm{~mm}$ is a safe option, but requires a standardized 
pathway in diagnosis. Our pathway has shown good reliability and security profile to be applied in a multicenter management for small scrotal masses. Our study has shown the reliability of the diagnostic-therapeutic pathway in the management of single testicular masses. The higher incidence of benign lesions in $60 \%$ of patients makes testis-sparing surgery a reliable option, where orchiectomy could be considered an overtreatment.

\section{REFERENCES}

1. Ates F, Malkoc E, Zor M, et al. Testis-sparing surgery in small testicular masses not suspected to be malignant. Clin Genitourin Cancer. 2016; 14:e49-53.

2. Shilo Y, Zisman A, Raz O, et al. Testicular sparing surgery for small masses. Urol Oncol. 2012; 30:188-91.

3. Brunocilla E, Gentile G, Schiavina R, et al. Testis-sparing surgery for the conservative management of small testicular masses: an update. Anticancer Res. 2013; 33:5205-10.

4. Palermo G, Antonucci M, Recupero SM, et al. Focal surgery in testis cancer: current state of the art. Urologia 2013; 80:290-6.
5. Galosi AB, Lacetera V, Muzzonigro G. Clinica delle malattie testicolari di interesse ecografico. Urologia 2008; 75,4, S12: 59-66.

6. Sbrollini G, Mazzaferro D, Ettamimi A, et al. Diagnostic-therapeutic pathway for small lesions of the testis. Arch Ital Urol Androl. 2014; 86:397-9

7. Fabiani A, Filosa A, Pieramici T, Mammana G. Testicular nodules suspected for malignancy. Does the pathologist make the difference for organ-sparing surgery? Anal Quant Cytopathol Histpathol. 2015; 37:147-52.

8. Steiner H, Höltl L, Maneschg C, et al. Frozen section analysisguided organ-sparing approach in testicular tumors: technique, feasibility, and long-term results. Urology. 2003; 62:508-13.

9. Friend J, Barker A, Khosa J, Samnakay N. Benign scrotal masses in children: some new lessons learned. J Pediatr Surg. 2016; 51:1737-42.

10. Martino P, Galosi AB, Bitelli M, et al. Imaging Working Group Societa Italiana Urologia and Societa Italiana Ecografia Urologica Andrologica Nefrologica. Practical recommendations for performing ultrasound scanning in the urological and andrological fields. Arch Ital Urol Androl. 2014; 86:56-78.

\section{Correspondence}

Andrea B Galosi, MD

galosiab@yahoo.it

Paola Fulvi, MD, Resident in Urology

paola.fulvi19@gmail.com

Luca Leone, Resident in Urology

lucaleone85@virgilio.it

Clinica Urologica, Dipartimento Science Cliniche e

Odontostomatologiche,Università Politecnica delle Marche,

AO Ospedale Riuniti, Ancona, Italy

Andrea Fabiani, MD Urologist

andreadoc1@libero.it

Lucilla Servi, MD Urologist

UOC Urologia Macerata, Area Vasta 3, ASUR Marche, Macerata, Italy

Alessandra Filosa, MD Pathologist

UOC Anatomia Patologica, Area Vasta 3, ASUR Marche, Italy

Angelo Marronaro, MD Urologist

Enrico Caraceni, MD Urologist

ecarace@libero.it

UOC Urologia, Civitanova Marche, Area Vasta 3, ASUR Marche,

Civitanova Marche, Italy

Rodolfo Montironi, MD, Pathologist

r.montironi@univpm.it

UOC Anatomia Patologica, Università Politecnica delle Marche, Ancona, Italy 